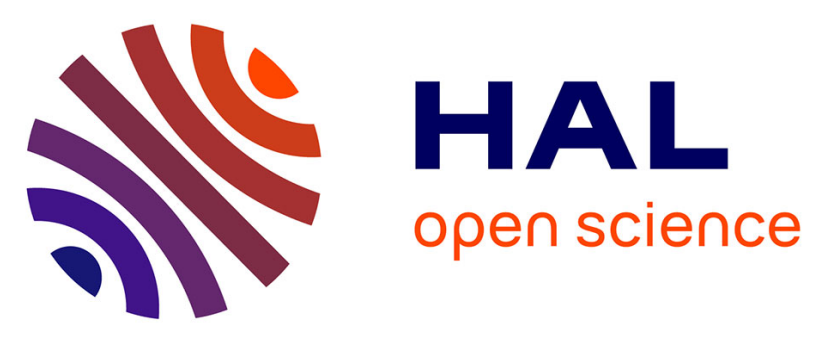

\title{
Practical comparisons of EIT excitation protocols with applications in high-contrast imaging
}

Chunhui Dang, Cédric Bellis, Mathieu Darnajou, Guillaume Ricciardi, Saba Mylvaganam, Salah Bourennane

\section{To cite this version:}

Chunhui Dang, Cédric Bellis, Mathieu Darnajou, Guillaume Ricciardi, Saba Mylvaganam, et al.. Practical comparisons of EIT excitation protocols with applications in high-contrast imaging. Measurement Science and Technology, 2021, 32 (8), pp.085110. 10.1088/1361-6501/abe742 . hal-03370772

\section{HAL Id: hal-03370772 \\ https://hal.science/hal-03370772}

Submitted on 8 Nov 2021

HAL is a multi-disciplinary open access archive for the deposit and dissemination of scientific research documents, whether they are published or not. The documents may come from teaching and research institutions in France or abroad, or from public or private research centers.
L'archive ouverte pluridisciplinaire HAL, est destinée au dépôt et à la diffusion de documents scientifiques de niveau recherche, publiés ou non, émanant des établissements d'enseignement et de recherche français ou étrangers, des laboratoires publics ou privés. 


\title{
Practical comparisons of EIT excitation protocols with applications in high-contrast imaging
}

\author{
Chunhui Dang ${ }^{1,2}\left({ }^{\circ}\right.$, Cédric Bellis ${ }^{3}$, Mathieu Darnajou ${ }^{1,2}\left(\mathbb{D}\right.$, Guillaume Ricciardi ${ }^{1}$, \\ Saba Mylvaganam ${ }^{3}$ and Salah Bourennane ${ }^{4}$ \\ ${ }^{1}$ CEA-DES-IRESNE-DTN, CEA Cadarache, Saint-Paul-lez-Durance, France \\ ${ }^{2}$ Institut Fresnel, CNRS, Centrale Marseille, Aix Marseille Univ, Marseille, France \\ ${ }^{3}$ Aix Marseille Univ, CNRS, Centrale Marseille, LMA UMR 7031, Marseille, France \\ ${ }^{4}$ Faculty of Technology, University College of Southeast Norway, Porsgrunn, Norway \\ E-mail: dang.chunhui@ centrale-marseille.fr
}

\begin{abstract}
In the field of electrical impedance tomography (EIT), numerous studies have aimed at the optimal excitation/ measurement strategies for improving conductivity distribution imaging, particularly in the applications involving highly contrasted materials. These studies focus on the conditions to be imposed on the currents fed into the electrodes and on their measurement counterparts, while making use of various quantitative optimal criteria. While most EIT systems rely on a sequential excitation at neighboring electrodes with measurements at the remaining ones, some alternative excitation strategies, or protocols, have also proven to be effective and easy to implement using modern hardware. In this context, the present study aims at confronting some of the predominant EIT excitation protocols on a practical system that is dedicated to the imaging of media with highly contrasted material components, i.e. with large variations of the conductivity field. More specifically, the so-called adjacent, opposite, full-scan and trigonometric excitation strategies are considered here and assessed on a number of criteria, which are: complexity of a practical implementation, number of independent measurements, amplitude of the measured responses, sensitivity distribution and quality of the final reconstructed images. For each of these excitation protocols, numerical simulations and static experiments with test objects placed in the EIT sensor considered are carried out using various conductivity profiles, while reconstructed images are evaluated both qualitatively and qualitatively. Our results highlight the preeminence of the full-scan and trigonometric strategies, which are characterized by high response signals and satisfying overall sensitivities. Moreover, for the studied configurations in both numerical simulations and static experiments, the full scan and trigonometric strategies lead to improved contrasts in the reconstructed images of the phases distribution.
\end{abstract}

Keywords: electrical impedance tomography, optimal excitation strategies, acquisition rates, response amplitude, sensitivity distribution, image reconstruction 


\section{Introduction}

To determine the constitutive electrical properties of a body, i.e. its internal conductivity or permittivity distribution, electrical impedance tomography (EIT) is a well-known technique applicable both to 2D and 3D configurations. With the advantages of non-intrusiveness, low cost and high temporal resolution, for time-dependent configurations, EIT has been successfully applied in medical imaging, multiphase flow studies or process monitoring, in which, very often, the conductivity profile exhibits a high contrast. In instrumentation for the nuclear industry, more specifically, there is a great need to investigate heat transfer behaviors and possible flow instabilities in reactor thermo-hydraulics, see [1], and EIT has shown to be a valuable technique, which enables real-time online monitoring.

In EIT, the current pattern imposed on the discrete set of electrodes may be chosen arbitrarily. Yet, there has been an intensive search for optimal patterns, starting in the field of medical imaging in the 1990s. Gisser et al [2] introduced the concept of distinguishability as a quantitative criterion to evaluate the quality of a current pattern. It is defined as the quantity $\delta=\left\|V-V_{0}\right\|_{L^{2}}^{2} /\|I\|_{L^{2}}^{2}$, where $V$ and $V_{0}$ are the boundary voltage measurements associated with the studied and reference media, given the current excitation $I$. Cheney and Isaacson [3] proved analytically that the so-called trigonometric pattern maximizes $\delta$. However, the use of an alternative criterion may lead to a different optimal current pattern. For example, if the total injected current is constrained, then it has been shown in [4] that the optimal current pattern turns out to be the so-called pair drive pattern. Furthermore, Demidenko et al [5] deployed a criterion that quantifies the accuracy of the reconstructed tomographic images, based on the total variance of the estimated resistance (or conductance) matrix, and developed an adaptive approach with real-time updating of the current pattern. The optimal current patterns described above have been investigated in depth mathematically. Yet, to our knowledge, there is a lack of a systematic comparison of these physical and numerical models using actual experimental data and indicators of the quality of reconstructed images, which is the ultimate goal of tomography. Clearly, there does not seem to be a consensus on the optimal current pattern to be used, but most of the practical systems in use employ the so-called adjacent pattern [6-8] with the advantage of easy implementation, although it is considered to be detrimental to distinguishing changes in internal conductivity distributions [9]. In this context, this article aims at confronting some of the predominant EIT excitation protocols on a practical system that is dedicated to the imaging of media with highly contrasted material components.

Considering, modern EIT architectures, it is easy in practice to switch from current to voltage excitations, so that, here, we will be investigating a number of excitation strategies, in a broad sense. Among the preeminent excitation strategies available and easily implementable with up-to-date hardware, the so-called opposite, full-scan [10, 11], and trigonometric strategies [3] have shown satisfying performances. Yet, they have their own pros and cons but lack exhaustive comparisons in practical set-ups. To our knowledge, only the adjacent and opposite strategies were compared in terms of reconstruction performances in Stephenson et al [12]. In the present study, the adjacent, opposite, full-scan and trigonometric strategies are under investigation. Our objective is to provide a comprehensive comparison of them, in terms of (a) feasibility of the hardware implementation, (b) number of independent measurements, (c) amplitude of the measurement responses, (d) sensitivity distribution, and (e) quality of the reconstructed images.

The four excitation strategies considered are introduced in section 2 with a brief review of EIT hardware designs followed by a short description of their implementation using modern hardware. The number $N_{m}^{i}$ of independent measurements accessible in each strategy is discussed, as it determines the spatial resolution of the reconstructed images produced and might not be necessarily equal to the number $N_{m}$ of measurements. In section 3, robustness to measurement noise is evaluated through computation of the mean and standard deviation of synthetic boundary measurements. In section 3.4, the effectiveness of each internal region contribution to the overall measurement signal is assessed numerically through sensitivity maps for each strategy. Note that Kauppinen et al [13] visualized such sensitivity distributions for the adjacent and opposite strategies, and also the so-called cross and adaptive ones, but it was for single excitation patterns rather than for the global excitation strategies that we consider here. In section 4 we focus finally on reconstructed images. Various iterative and non-iterative algorithms have been proposed to reconstruct images in EIT. Such algorithms have been thoroughly investigated and compared in the context of high contrast imaging in chemical engineering or process tomography, see e.g. $[14,15]$. Here, we focus specifically on the role of the excitation strategy itself on the reconstructed images. Therefore, in the present study, the images obtained for the excitation strategies considered are compared numerically for different reference conductivity profiles. Different criteria are proposed in section 4 for quantitative comparisons. Moreover, two types of regularizations are applied in the reconstruction process, namely the Tikhonov regularization and the total variation (TV) regularization. Lastly, section 4.3 presents and compares a set of tomographic images corresponding to experimental static tests carried out in the laboratory of analytical thermohydraulics and hydromechanics of core and circuits (LTHCs) with still water and plastic rod(s) as the object(s) to be reconstructed.

\section{EIT protocols}

\subsection{Setting}

Consider the electric conductivity problem in a two- or threedimensional domain $\Omega$, which we consider to be respectively 
circular or cylindrical for simplicity. Here, the medium is assumed to be isotropic. From Maxwell's equations, the electrostatic potential $u$ within $\Omega$ is governed by

$$
\nabla \cdot(\sigma(\boldsymbol{x}) \nabla u(\boldsymbol{x}))=0 \quad(\boldsymbol{x} \in \Omega)
$$

where $\sigma$ is the scalar electric conductivity distribution. Moreover, the medium is assumed to be piecewise homogeneous as a background containing a subset $\Omega_{\mathrm{i}}$ characterized by a significantly lower conductivity.

The field equation (1) has to be complemented by boundary conditions. To do so, an even number $\ell$ of identical and uniformly spaced electrodes $e_{k} \subset \partial \Omega$ are used to apply an excitation current (resp. voltage) and measure the resulting voltage (resp. current). The electrodes are assumed to be perfectly conducting so that the potential $\left.u\right|_{e_{k}}$ at each electrode is constant. At a given electrode $k$, one considers either one of the following conditions:

$$
\begin{array}{ll}
\int_{e_{k}} \sigma \nabla u \cdot \boldsymbol{n} \mathrm{d} s=I_{k} & \text { for current excitation, } \\
\left.u\right|_{e_{k}}=V_{k} & \text { for voltage excitation, }
\end{array}
$$

where $I_{k}$ (resp. $V_{k}$ ) is the imposed current (resp. voltage) applied on $k$ th electrode. In (2) one uses implicitly the socalled shunt electrode model, see e.g. [16], with $\boldsymbol{n}$ being the unit outward normal vector on $\partial \Omega$. Moreover, the interstitial spaces in between electrodes are assumed to be isolated, so that it holds $\sigma \nabla u \cdot \boldsymbol{n}=0$ on $\partial \Omega \backslash \cup_{k} e_{k}$. For all excitation patterns, the excitation currents or voltages are assumed to sum up to zero. Lastly, the boundary measurements are taken simultaneously at all of the $\ell$ electrodes, including the excitation carrying electrode(s). Note that, in some applications, the measurements at the excitation carrying electrode(s) are discarded in post-processing to reduce the possible influence of contact impedance.

\subsection{Excitation strategies}

EIT architectures can be classified into pair drive and multiple drive systems depending on the number of excitation carrying electrodes that are active at a given time. In a pair drive system, the excitation is applied through a pair of source and drain electrodes. In a multiple drive system, all of the electrodes are active simultaneously. In any case, the specific form of the boundary condition considered defines an excitation pattern, which is ultimately used to compute a tomographic image. A given series of patterns can be applied sequentially to different electrodes to form the excitation strategy. The detailed introduction is presented hereafter.

\subsubsection{Pair drive systems}

2.2.1.1. Adjacent strategy. It is defined by the pattern where the excitation current is injected at two neighboring electrodes. In the adjacent strategy, the current is switched to the next pair of neighboring electrodes until all the possible neighboring pair have been considered. The number $N_{e}$ of excitation patterns is equal to the number of electrodes, i.e. $N_{e}=\ell$, yielding a number of measurements $N_{m}=\ell^{2}$. The main drawback of the adjacent strategy is the expected non-uniformity of the current distribution within the domain, with the current density being maximized in a neighborhood of the excitation electrodes and much lower at distant electrodes, thus leading to measurements of low amplitude at the latter, which would therefore be more susceptible to noise pollution.

2.2.1.2. Opposite strategy. It considers current injection at pairs of opposite electrodes, with $N_{e}=\ell / 2$ possible excitation patterns, which gives $N_{m}=\ell^{2} / 2$ measurements. The latter value is half this associated with the adjacent strategy, which can be expected to be detrimental to the images to be reconstructed. Nonetheless, the signal amplitudes acquired at distant electrodes can be expected to be larger in the opposite strategy compared to the adjacent one, as the maximal distance between excitation and measurement electrodes being approximately reduced by a factor $\sqrt{2}$ for the former.

2.2.1.3. Full-scan strategy. Here, all electrodes are sequentially selected to be source and drain until all possible pairs of electrodes have been formed, giving $N_{e}=\ell(\ell-1) / 2$ and $N_{m}=\ell^{2}(\ell-1) / 2$. This strategy includes the excitation patterns of the adjacent and opposite strategies, among others, thus leading to comparatively longer acquisition times. Indeed, it respectively involves $(\ell-1) / 2$ and $(\ell-1)$ times more patterns than the latter.

2.2.2. Multiple drive systems. Among the possible excitation strategies applicable to multiple drive EIT systems, we only focus here on the following one which seems to be favored by previous studies.

2.2.2.1. Trigonometric strategy. This strategy has been introduced in the search for an optimal excitation pattern and it has been shown to maximize a distinguishability criterion, see [3]. In each excitation pattern, the current at the electrodes $e_{k}$, for $k=1, \ldots, \ell$, is constructed from a cosine or sine function of varying angular frequency $\omega_{j}=2 \pi j / \ell$, as

$$
I_{k, j}= \begin{cases}I \cos \left(k \omega_{j}\right) & \text { with } 1 \leqslant j \leqslant \ell / 2, \\ (-1)^{k+1} I \sin \left(k \omega_{j}\right) & \text { with } \ell / 2<j \leqslant(\ell-1),\end{cases}
$$

where $I$ is a fixed amplitude. The definition (3) of $I_{k, j}$ is to be used in the boundary condition (2) and it can be adapted to the case of voltage excitations. This defines $N_{e}=(\ell-1)$ different patterns that lead to $N_{m}=\ell(\ell-1)$ measurements overall.

To summarize, the schematics of the four excitation strategies considered are shown in figure 1. Compared to the other strategies, the trigonometric one requires simultaneous excitation of multiple sources, which is more demanding in practice and increases the complexity of the EIT system. Yet, recent hardware and software developments make its implementation possible at reasonable costs; see [17]. 


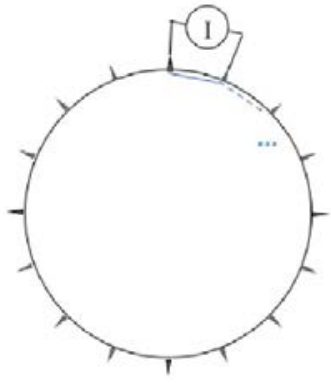

(a) Adjacent excitation

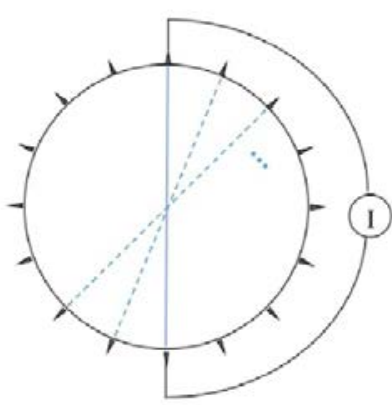

(b) Opposite excitation.

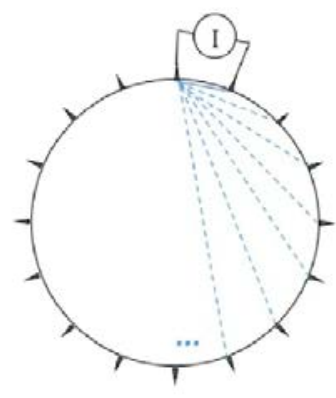

(c) Full-scan excitation.

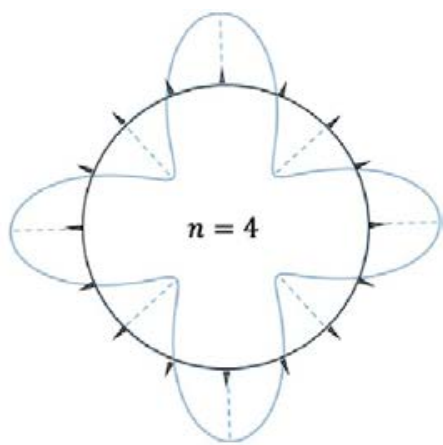

(d) Trigonometric excitation.

Figure 1. Schematics of the four excitation strategies considered. In (a)-(c) the blue dotted lines indicate the pattern of electrode pairs that are activated in the excitation. In (d) the trigonometric function with a frequency $j=4$ is plotted (solid blue line), giving the excitation amplitude at each electrode.

\section{Practical implementations}

\subsection{EIT architectures}

In this section, we describe typical EIT architectures and provide some estimations of the representative acquisition frame rates for each strategy (a frame being composed of the acquisitions associated with all of its excitation patterns) with $\ell=16$ electrodes.

3.1.1. Conventional systems. For pair drive systems, a multiplexer is generally used to switch a single pair of source and drain electrodes. For optimal performances it can be mounted on a printed circuit board, together with the excitation and measurement electronic modules, as well as, for some systems, a signal filter and amplifier. In such settings, using commercial multiplexer commonly available, the acquisition rate could typically reach a few hundreds frames per second (fps), see e.g. $[6,18,19]$. For these systems, the transient phases during multiplexing have been shown to have an effect on the measurements, in particular due to the contact impedance of the electrode-electrolyte interface; see [16]. Therefore, the measurements performed at the excitation carrying electrodes are generally discarded. Note that, it has been evidenced in [11] that the residual error induced by multiplexing could be neglected when employing an appropriate switching method and large electrode area. In the present study, all measurements are taken into account when considering pair drive excitations. For the multiple drive trigonometric strategy, then multiplexing is not needed since each electrode is directly connected to an individual electrical source whose amplitude varies with the excitation pattern. Yet, with the requirement to control all of these individual sources, the system becomes more complex.

In the context considered, the ProMET system of [11] is now taken as a prototypical example of EIT system and the associated frame rates are provided for the various excitation strategies, see table 1 . The signal excitation and acquisition in ProMET are both realized with the National Instrument (NI) PXI-6368e card [20]. It has four analogue output (AO)
Table 1. Estimated acquisition frame rates (fps) for the excitation strategies considered and using an EIT system with 16 electrodes.

\begin{tabular}{lccll}
\hline Strategies & Adjacent & Opposite & Full-scan & Trigonometric \\
\hline $\begin{array}{l}\text { Conventional } \\
\text { system [11] }\end{array}$ & 1560 & 3120 & 208 & 1664 \\
Fast system [17] & 31250 & 62500 & 3906 & 31250 \\
\hline
\end{tabular}

channels at a sampling rate up to $3.33 \mathrm{MHz}$, and 16 differential Analogue Input (AI) channels up to $2 \mathrm{MHz}$. Moreover, it features a 16 ports multiplexer to switch the AO signals to selected pair of electrodes. Using ProMET, the typical frame rate for the full-scan strategy is $208 \mathrm{fps}$ considering a compromise between frame rate and measurement accuracy. Correspondingly, the typical frame rates for the adjacent and opposite strategies are $1560 \mathrm{fps}$ and $3120 \mathrm{fps}$ (recall the number of excitation patterns of various strategies in section 2.2.1), respectively. Lastly, for the trigonometric strategy, three more PXIe cards are required to drive excitation signal at each electrode, which therefore bear an extra cost. The resulting frame rate is estimated at $1664 \mathrm{fps}$.

3.1.2. Fast systems. The logic of EIT hardware design has been mature for decades now and recent advances have focused on greater accuracy, faster acquisitions and the ability to apply parallel multi-frequency excitations [21]. Recently, an innovative concept called ONe Excitation for Simultaneous High-speed Operation Tomography (ONE-SHOT) has been proposed in [22], in which frequency-division multiplexing (FDM) is used to discriminate some superimposed signals at multiple frequencies, giving access to boundary measurements of multiple excitation patterns simultaneously. It is applicable to both pair drive and multiple drive systems without multiplexing.

The ONE-SHOT method has been implemented in [17] using the NI CRIO-9039 controller [23]. Benefiting from a field programmable gate array (FPGA), they developed an ultra-high speed EIT system implementing the full-scan strategy. A total number $N_{d}=$ nextpower $2\left(2 N_{e}\right)$ of data points 
Table 2. Number $N_{m}^{i}$ of independent measurements and redundancy ratio relatively to the number $N_{m}$ of measurements for the various strategies, considering an EIT system with 16 electrodes.

\begin{tabular}{lllll}
\hline Strategies & Adjacent & Opposite & Full-scan & Trigonometric \\
\hline$N_{m}^{i}$ & 120 & 92 & 120 & 240 \\
$N_{m}^{i} / N_{m}$ & 0.47 & 0.72 & 0.06 & 1 \\
\hline
\end{tabular}

were acquired at the minimum for each frame in order to discriminate the signals of $N_{e}$ excitation patterns using fast Fourier transform under the Nyquist criterion. The controller has eight ports for AI/AO modules with a sampling rate up to $1 \mathrm{MHz}$, leading to the maximum frame rate of $10^{6} / N_{d}$ fps. The estimated frame rates are provided in table 1 . Still, it is underlined that the trigonometric strategy requires 14 extra current sources for a fast system as in a conventional system.

\subsection{Number of independent measurements}

For an EIT system with $\ell$ electrodes, Breckon and Pidcock [24] showed that, for any pair drive strategy, the number $N_{m}^{i}$ of independent measurement is at most equal to $\ell(\ell-1) / 2$. Yet, the opposite strategy is such that $N_{m}^{i}=\ell(3 \ell-2) / 8$ because it makes use of a relatively limited number of excitation patterns. Particularizing in the case of an EIT system with 16 electrodes then table 2 provides the number $N_{m}^{i}$ of independent measurements and the ratio with the number $N_{m}$ of measurements for the various excitation strategies considered. This ratio is called the redundancy ratio, as it quantifies the redundancies in the measurements, with the value 1 being attained if they are all independent. Conversely a small ratio characterizes large measurements redundancies, which may be a factor to be considered if memory and computational power are an issue.

\subsection{Statistics of measurement amplitudes}

Due to the ill-posedness of the EIT inverse problem, even a small measurement noise can lead to large discrepancies in the reconstructed images. Thus, in practice, additionally to the number $N_{m}^{i}$ of independent measurement discussed previously, it is also insightful to evaluate the statistical distributions of the measurement amplitudes at different electrodes for the various excitation strategies. These are investigated here based on numerical simulations. The finite-element based open source software EIDORS [25], is used to build an EIT model for the homogeneous background conductivity and compute a set $\boldsymbol{V}$ of boundary measurements for each strategy. The domain is set to be a disk with unit radius and 16 evenly spaced electrodes with peripheral length equal to 0.16 , which coincides with the experimental EIT system considered hereafter.

In a preliminary stage, we assessed the effect of the mesh size and structure on both the forward and inverse solutions:
Table 3. Mean and standard deviation of the boundary measurements for the various excitation strategies and a 16 electrodes EIT system.

\begin{tabular}{lcccc}
\hline Strategies & Adjacent & Opposite & Full-scan & Trigonometric \\
\hline Mean (V) & 0.1931 & 0.3220 & 0.2817 & 0.8067 \\
Standard & 0.3915 & 0.2668 & 0.3130 & 0.3352 \\
deviation (V) & & & & \\
\hline
\end{tabular}

meshes with 256, 1024, 2304, and 8791 elements are considered, and taking the case with the finest mesh as a reference, the mean relative errors on the boundary measurements for the other cases were found to be $1.4 \%, 0.19 \%$, and $0.1 \%$, respectively. This is as expected and agrees with the results reported in Darnajou [26]. Therefore, the accuracy of the forward solution is only marginally improved with over 1024 mesh elements, as is in turn the resolution of the reconstructed images. Here, the mesh we consider (a triangular mesh with 8791 elements) is deemed sufficiently fine to give accurate forward solutions and, as a consequence, converged reconstructions. As to the resolution of the reconstructed images, once converged on a sufficiently fine mesh, it is mainly dependent on the information encapsulated in the boundary measurements, which depend on the number of electrodes and the choice of the excitation protocols, an issue that is the main focus of the present study.

For each strategy one considers a current source of unit amplitude. The mean (average amplitude) and the standard deviation (spatial variability) computed for each measurement set $\boldsymbol{V}$ are reported in table 3 .

For pair drive systems, the adjacent strategy is characterized by the lowest mean and the highest standard deviation, and conversely for the opposite strategy. The full-scan is associated with intermediate values. Indeed, the adjacent strategy has much lower measurement amplitudes at electrodes away from the source electrodes, which are more distant on average compared to the opposite strategy. The full-scan strategy contains the excitation patterns of the two previous strategies among others. For multiple drive systems, considering the trigonometric strategy yields measurements of a much larger amplitude in average compared to pair drive systems since it inputs more power into the system with all electrodes being active in each pattern. Moreover, the measurements standard deviation is comparable to the full-scan strategy.

\subsection{Sensitivity distribution}

Given an EIT system, a localized conductivity perturbation $\delta \sigma$ within the probed medium can induce a perturbation $\delta u$ of the electric potential, which can in turn be measured on the boundary. For a certain excitation strategy, the greater the perturbation $\delta u$, the more sensitive the system under consideration is. Considering a finite-element discretization of 

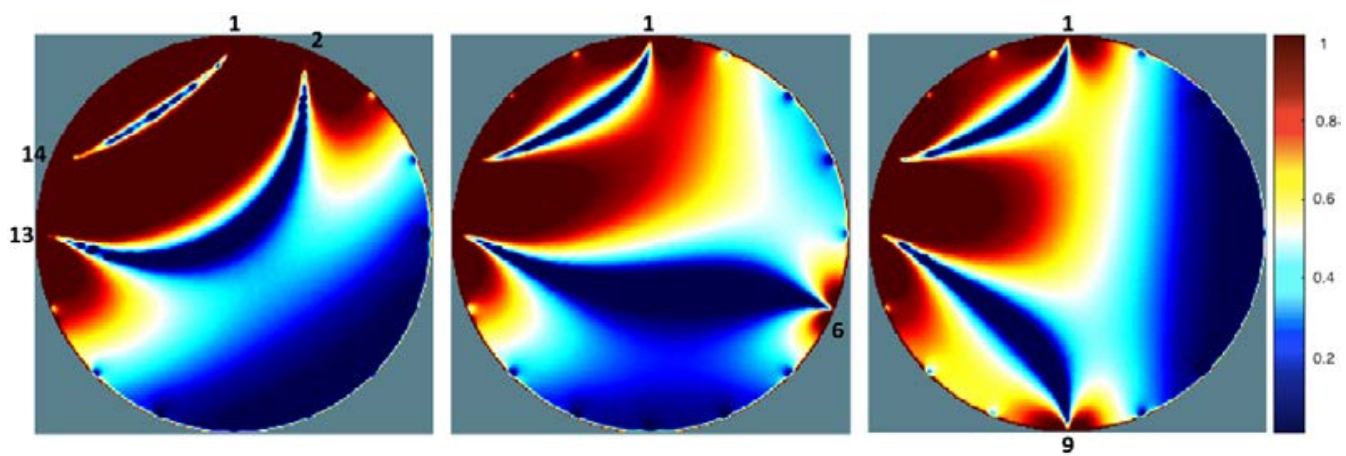

Figure 2. Logarithm of the absolute elementary sensitivity fields $s_{(i, k) j}$ after normalization for the cases with source/drain at electrode pair $(1,2),(1,6)$ and $(1,9)$, respectively, and measurement at electrode pair $(13,14)$.

Table 4. Mean and standard deviation of the sensitivity distributions for the various excitation strategies and a 16 electrodes EIT system; see also figure 3.

\begin{tabular}{lcccc}
\hline Strategies & Adjacent & Opposite & Full-scan & Trigonometric \\
\hline Mean & 0.8090 & 1.3406 & 2.0921 & 1.3810 \\
Standard deviation & 0.4636 & 0.2816 & 0.3234 & 0.5166 \\
\hline
\end{tabular}

the background domain, with piecewise-constant conductivity, and a perturbation $\sigma_{j} \rightarrow\left(\sigma_{j}+\delta \sigma_{j}\right)$ in the $j$ th element, then excitation is driven at electrode pair $i$ and the potential perturbation $V_{i k} \rightarrow\left(V_{i k}+\delta V_{i k}\right)$ is measured at electrode pair $k$. Note that the labelling $(i, k)$ of excitation and measurement electrode pairs is a numbering specific to each excitation strategy that is kept implicit here to avoid technicalities. In order to characterize the imaging capabilities of the EIT system, one considers the ratio

$$
s_{(i, k) j}=\frac{\delta V_{i k}}{\delta \sigma_{j}}=\int_{j} \nabla u_{i}(\boldsymbol{x}) \cdot \nabla u_{k}(\boldsymbol{x}) \mathrm{d} \boldsymbol{x},
$$

The second equality uses the adjoint method, with the integral being carried over the $j$ th element, $u_{i}$ is the voltage field associated with $i$ th current pattern while $u_{k}$ is the voltage field computed using the $k$ th voltage measurement as an excitation current. The equation (4) quantifies the sensitivity of the $k$ th measurement in the $i$ th excitation pattern to a local conductivity perturbation in the $j$ th element. The complete set of sensitivities can be concatenated in the form of a Jacobian matrix $\boldsymbol{J}$.

An overall (discrete) sensitivity map $S_{j}=\sum_{i} \sum_{k} s_{(i, k) j}$ is defined as the element-wise summation of the sensitivities of all patterns in one given excitation strategy. Using the same finite element EIT model as in section 3.3, the overall sensitivity map $S_{j}$ is computed for each excitation strategies considered. First, figure 2 shows the logarithm of the absolute elementary sensitivity fields in (4) after normalization, for one single measurement at electrode pair $(13,14)$, and the excitation being applied at the electrode pair $(1,2),(1,6)$ and $(1,9)$, from the left to right panels respectively. As expected, the sensitivity is lowest in the regions that are the most distant from the excitation/measurement electrodes. However, these maps also highlight the, somehow counterintuitive, distribution of the sensitivity field with narrow 'valleys' of low sensitivity connecting the excitation and measurement electrodes. This intriguing behavior have already been described and we have discussed it more extensively in [26, 27].

Figure 3(a) plots the resulting overall sensitivity distribution $S_{j}$ along the domain diameter for the various excitation strategies considered. The computed fields $S_{j}$ are radially symmetric (local averaging have been performed to smooth out discretization effects), and these values are normalized in figure 3(b) relatively to the associated maximal sensitivity, which is naturally attained on the boundary. Some relevant statistics are reported in table 4.

From figure 3 and table 4, the adjacent strategy is characterized by the lowest overall sensitivity among the four strategies considered, with a $80 \%$ drop in sensitivity at the centre of the domain. The opposite strategy has a more homogeneous sensitivity distribution (small standard deviation) compared to the adjacent one. The full-scan strategy is characterized by the highest overall sensitivity values. Finally, the trigonometric strategy has relatively large sensitivity values but they significantly drop (by about 66\%) at the center of the probed domain.

\subsection{Practical implementation criteria}

To conclude the section on the practical aspects of the implementation of the four excitation strategies considered, figure 4 concatenates all of the quantitative criteria of the tables 1-4. With the exception of standard deviation criteria, which one generally intends to minimize, all of these criteria are increasing functions of performance. For a synthetic representation, these criteria are plotted after being each normalized by the largest value among the excitation strategies. Note that, according to the previous comment, reversed ranking is used for the standard deviation criteria. Such a representation highlights that defining what is an optimal strategies depends on which criteria are important in a practical implementation. For example, the trigonometric strategy satisfies well most criteria 


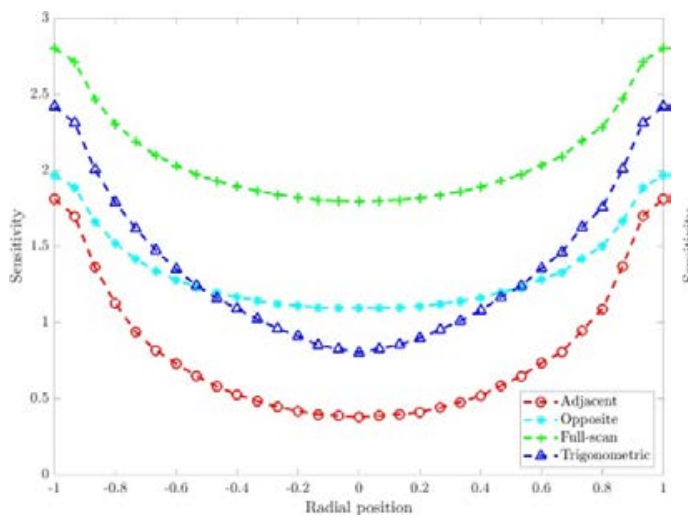

(a) Sensitivity distribution.

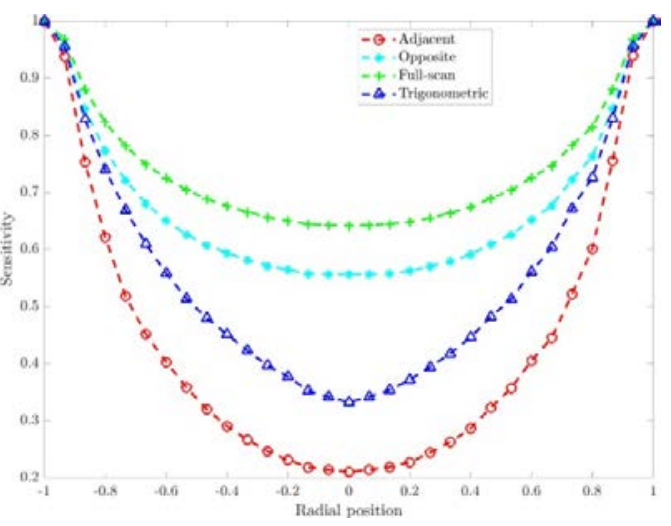

(b) Normalized distribution.

Figure 3. Locally averaged sensitivity distributions along one diameter for the excitation strategies considered.

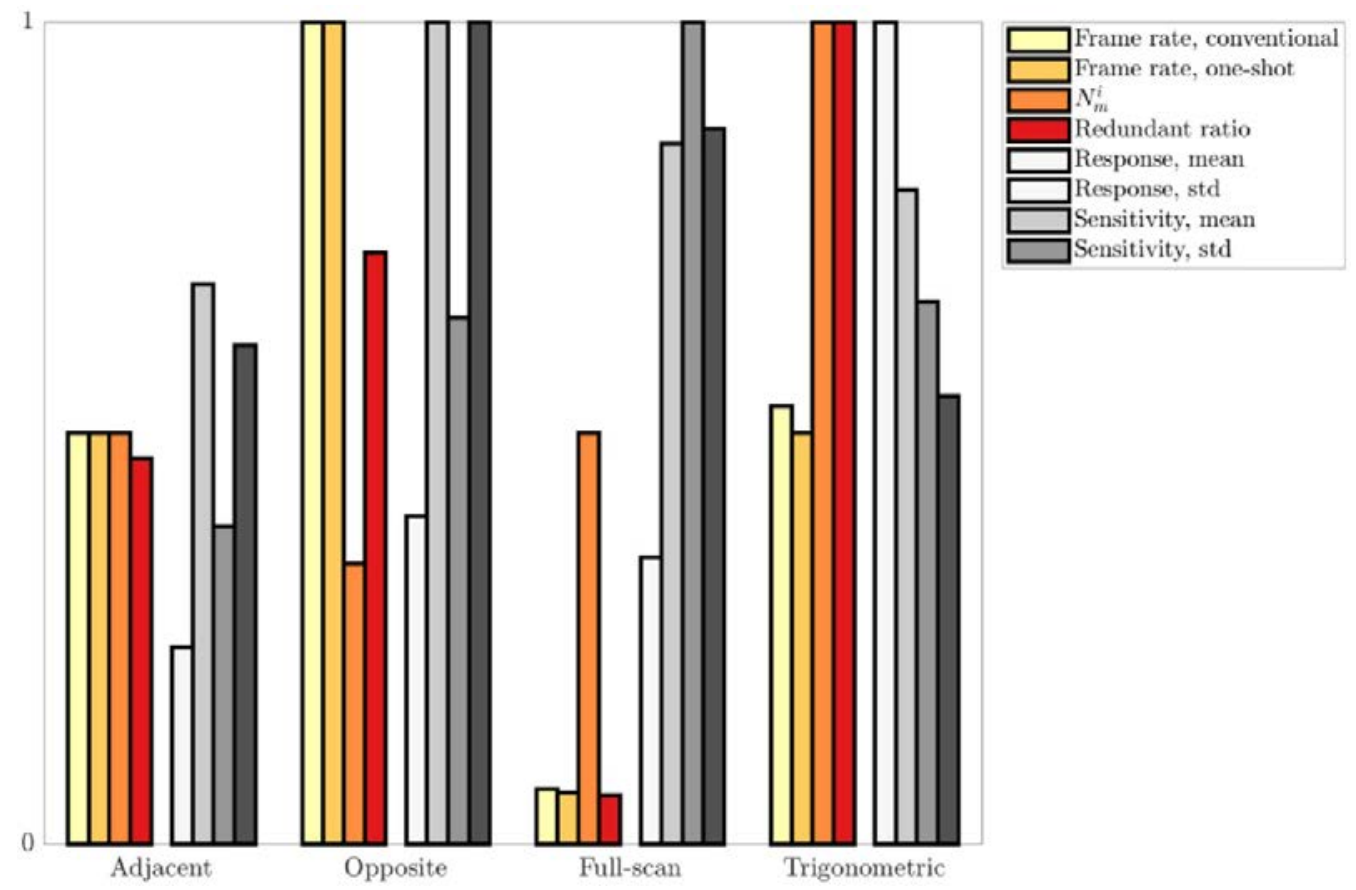

Figure 4. Practical implementation criteria, each normalized by the largest value among the four excitation strategies. The value 1 is associated with the best practical performance and low values indicate relatively lower-performing strategies.

on average. As for the full-scan, on the one hand it bears substantial acquisition costs but, on the other hand, it is characterized by high responses signals and sensitivity. In this context, we turn in the section 4 to a comparison of reconstructed images with some quantitative evaluations of their quality.

\subsection{Preliminary study on the fringe effect}

Reconstructions in a 3D volume can also be investigated, in particular to study the behavior of the electrical fields and the sensitivity distribution outside of the electrode plane, which are related to the so-called fringe effect [28]. Here, preliminary $2 \mathrm{D}$ simulations are performed, modeling a slice of a 3D domain, to investigate spreading effects in EIT when multiple planes of electrodes are employed. The open-source code FreeFem $++[29]$ is used to solve the forward EIT problem.

A number of studies have investigated the effects of socalled guarding electrodes on the potential and current fields distributions within the region of interest, see [30, 31]. The approach consists in employing extra planes of electrodes (referred to as the guarding electrodes) aside the main plane. The former can be either grounded or excited by the same voltage sources as the main electrodes [30]. In the figure 5, the domain of interest is set to be a rectangle with unit height and a width of 2 . On the top and bottom boundaries, three pairs of electrodes are considered, with a fixed length $L_{e}=0.2$ and 


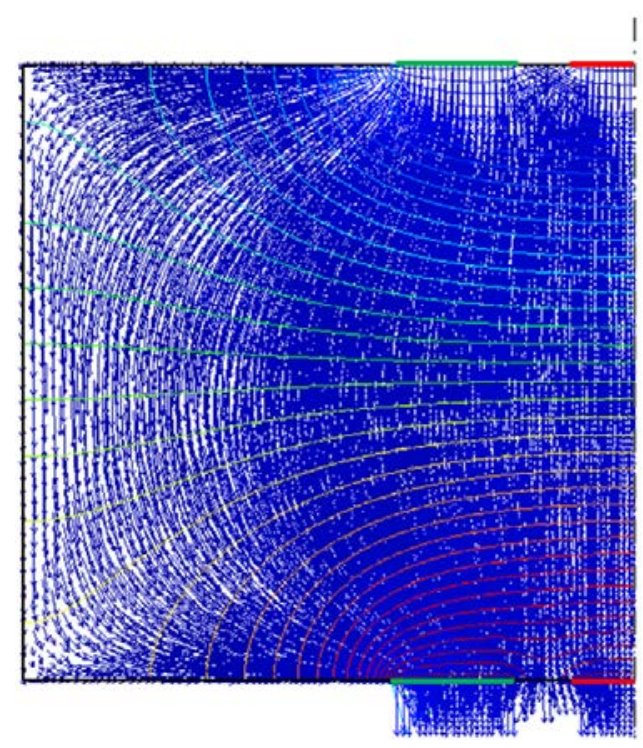

(a) $L_{d}=0.1$.
I

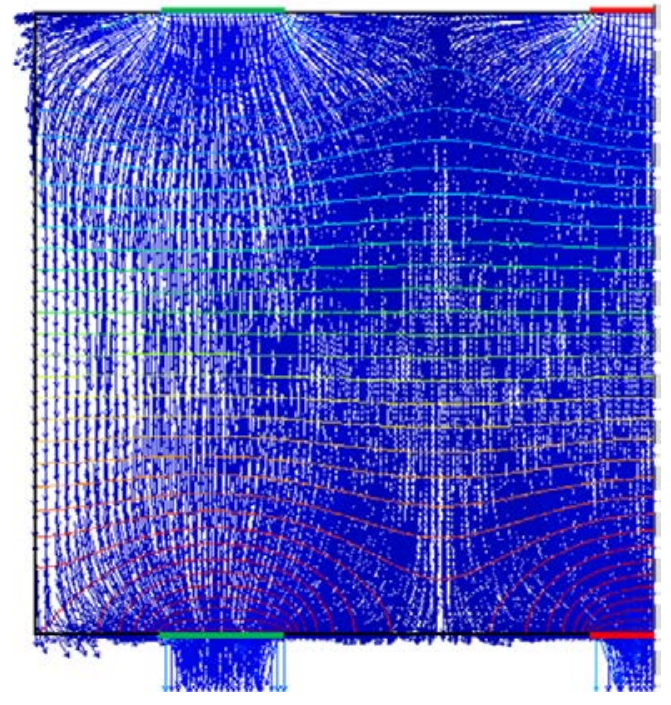

(b) $L_{d}=0.7$.

Figure 5. Current streamlines and equipotential lines within the domain of interest, for $L_{e}=0.2$ and $L_{d}=0.1,0.7$. The guarding electrodes (in green) are excited with the same voltage as the main plane electrodes (in red). Only half of the domain is shown as the configuration is symmetric.

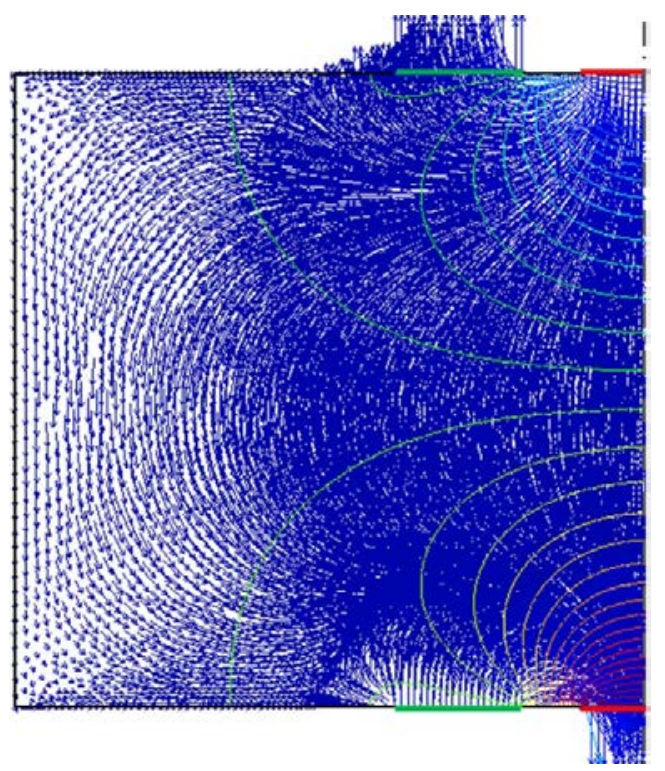

(a) $L_{d}=0.1$.

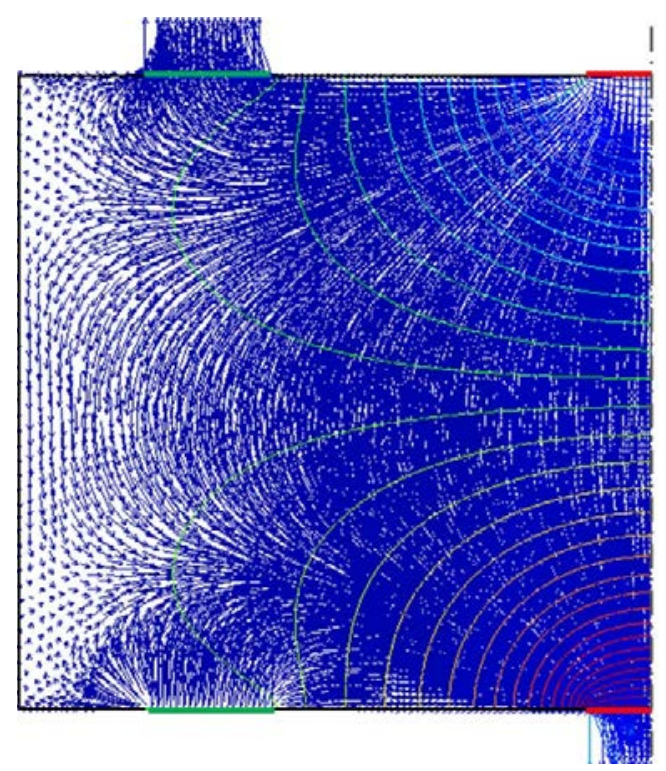

(b) $L_{d}=0.7$.

Figure 6. Current streamlines and equipotential lines within the domain of interest for $L_{e}=0.2$ and $L_{d}=0.1,0.7$. The guarding electrodes (in green) are grounded with zero potential. Only half of the domain is shown.

different distances $L_{d}$ between the guarding electrode plane (in green) and the main plane (in red). The rest of the domain boundary is insulated, i.e. with a zero current density condition. Voltage sources with amplitudes of $\pm 1 \mathrm{~V}$ are exerted on the main plane electrodes and the potential and current fields are computed and shown for $L_{d}=0.1$ and $L_{d}=0.7$. Only the left half of the domain is represented due to the symmetry of the configuration.
Figure 5 corresponds to the case where the guarding electrodes are excited by the same voltage as the main plane electrodes, while in figure 6 the former are grounded, i.e. with a zero potential condition. For comparison, a simulation result without guarding electrodes is reported in figure 7 , giving the current streamlines distribution when only a single pair of electrodes of length $L_{e}=0.2$ is considered. Comparing the fields in the main plane region in the figures 5 and 7 , it is 
Table 5. Geometric parameters defining the cases considered with the circular object(s) to be reconstructed.

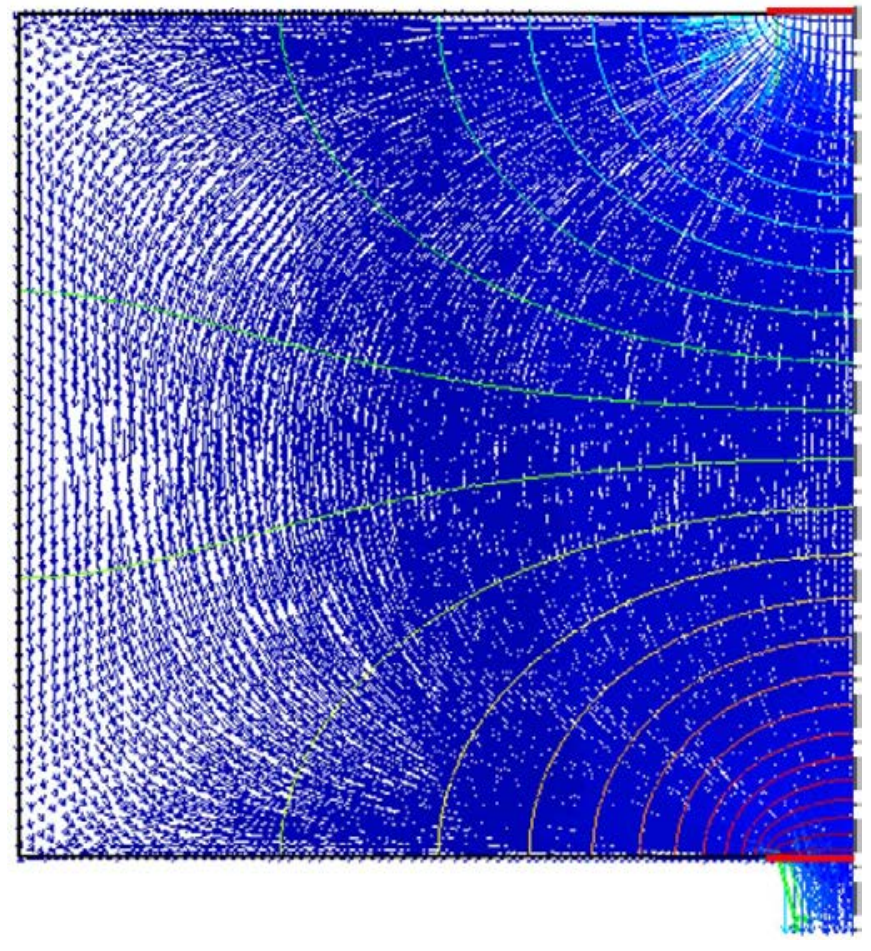

Figure 7. Current streamlines and equipotential lines within the domain of interest with a single pair of electrodes of length $L_{e}=0.2$. Only half of the domain is shown.

interesting to observe that spaced and excited guarding electrodes contribute to flatten the current streamlines compared to the case without guarding electrodes, which may in turn bring some benefits in diminishing the inhomogeneity of the 3D EIT sensitivity distribution. Such an effect is not reproduced with grounded guarding electrodes; see figure 6.

Although it has been argued in the literature that the use guarding electrodes does not fulfill the expectation that it reduces geometrical spreading effects, see e.g. [31], it may however provide some other advantages, such as a more homogeneous sensitivity distribution and stronger measurement signals. A comprehensive studies with full 3D simulations and comparisons with experiments are in demand for future researches, especially considering different excitation strategies on multiple plane of electrodes.

\section{Comparison of reconstructed images}

This section aims at a comparison of images reconstructed from measurements associated with the excitation strategies considered. To do so, the following quantitative criteria are introduced: global error in the identification of the conductivity field and geometrical errors in the reconstruction of the sought object(s) (area and position). Two regularized reconstruction methods are considered to make a sound comparison.

\begin{tabular}{|c|c|c|c|c|c|}
\hline Case & 1 & 2 & 3 & 4 & 5 \\
\hline$r$ & 0.2 & 0.7 & 0.2 & {$\left[\begin{array}{lll}0.25 & 0.15\end{array}\right]$} & {$\left[\begin{array}{lll}0.25 & 0.15\end{array}\right]$} \\
\hline$d$ & 0 & 0 & 0.7 & {$\left[\begin{array}{ll}0 & 0.7\end{array}\right]$} & {$\left[\begin{array}{ll}0.5 & 0.7\end{array}\right]$} \\
\hline
\end{tabular}

\subsection{Reconstruction methods}

The EIT inverse problem is solved in a discrete setting, i.e. using a finite element implementation with typical mesh size $h$, through the cost functional minimization process:

$$
\begin{aligned}
\boldsymbol{\sigma}_{\text {id }}^{h} & =\arg \min _{\boldsymbol{\sigma}^{h}} \mathcal{J}\left(\boldsymbol{\sigma}^{h}\right) \text { with } \\
\mathcal{J}\left(\boldsymbol{\sigma}^{h}\right) & =\frac{1}{2}\left\|\boldsymbol{V}^{h}\left(\boldsymbol{\sigma}^{h}\right)-\boldsymbol{V}^{h}\left(\boldsymbol{\sigma}_{\text {true }}^{h}\right)\right\|^{2}+\alpha \mathcal{R}\left(\boldsymbol{\sigma}^{h}, \boldsymbol{\sigma}_{\text {ref }}^{h}\right),
\end{aligned}
$$

where $\boldsymbol{V}^{h}\left(\boldsymbol{\sigma}_{\text {true }}^{h}\right)$ is a vector of discrete boundary measurements associated with an objective (discrete) conductivity distribution $\boldsymbol{\sigma}_{\text {true }}^{h}$ to be reconstructed, while $\boldsymbol{V}^{h}\left(\boldsymbol{\sigma}^{\boldsymbol{h}}\right)$ is a vector of simulated boundary measurements associated with a trial conductivity field $\boldsymbol{\sigma}^{h}$. Moreover, $\mathcal{R}$ is a regularization function, associated with the penalty coefficient $\alpha \in \mathbb{R}$ and, possibly, with a reference discrete conductivity field $\sigma_{\text {ref }}^{h}$. The minimizer $\boldsymbol{\sigma}_{\text {id }}^{h}$ gives the reconstructed image.

In the present study, two methods are considered: (a) the Newton's one-step error reconstructor (NOSER) method, see [32], which constrains the image amplitude and for which regularization is performed through penalization by the diagonal elements of the Jacobian matrix $\boldsymbol{J}$; (b) an iterative method with TV regularization, which tends to preserve discontinuities in the reconstructed conductivity distribution thus leading to reconstructions with sharp contrasts, see e.g. [33]. For various excitation strategies, a value of $\alpha$ is decided through the L-curve method in a preliminary analysis with a reference object.

\subsection{Image reconstructions from synthetic data}

Five different objective conductivity distributions $\sigma_{\text {true }}^{h}$ are considered in a finite element setting. The background medium is a disk with unit diameter endowed with conductivity $\sigma_{\text {true }}^{h}=1$, which contains one or two low conductivity objects, where $\sigma_{\text {true }}^{h}=0.1$. These objects are circular, with radius $r$ and distance $d$ to the origin, and the case defining values are reported in table 5. For such configurations, both the forward and inverse problems are solved using the finite-element software EIDORS (see section 3.3), with the two reconstruction methods described previously. The inverse computational model contains 1024 mesh elements, which is deemed sufficiently fine for the reconstructions to be converged, see also the discussion in section 3.3.

4.2.1. Reconstructed images. The obtained images, i.e. the reconstructed conductivity contrasts $\Delta \sigma_{\text {id }}^{h}=\left(\sigma_{\text {id }}^{h}-1\right)$, for the various excitation strategies considered are shown in figure 8 for both the NOSER method and TV regularization. In each 


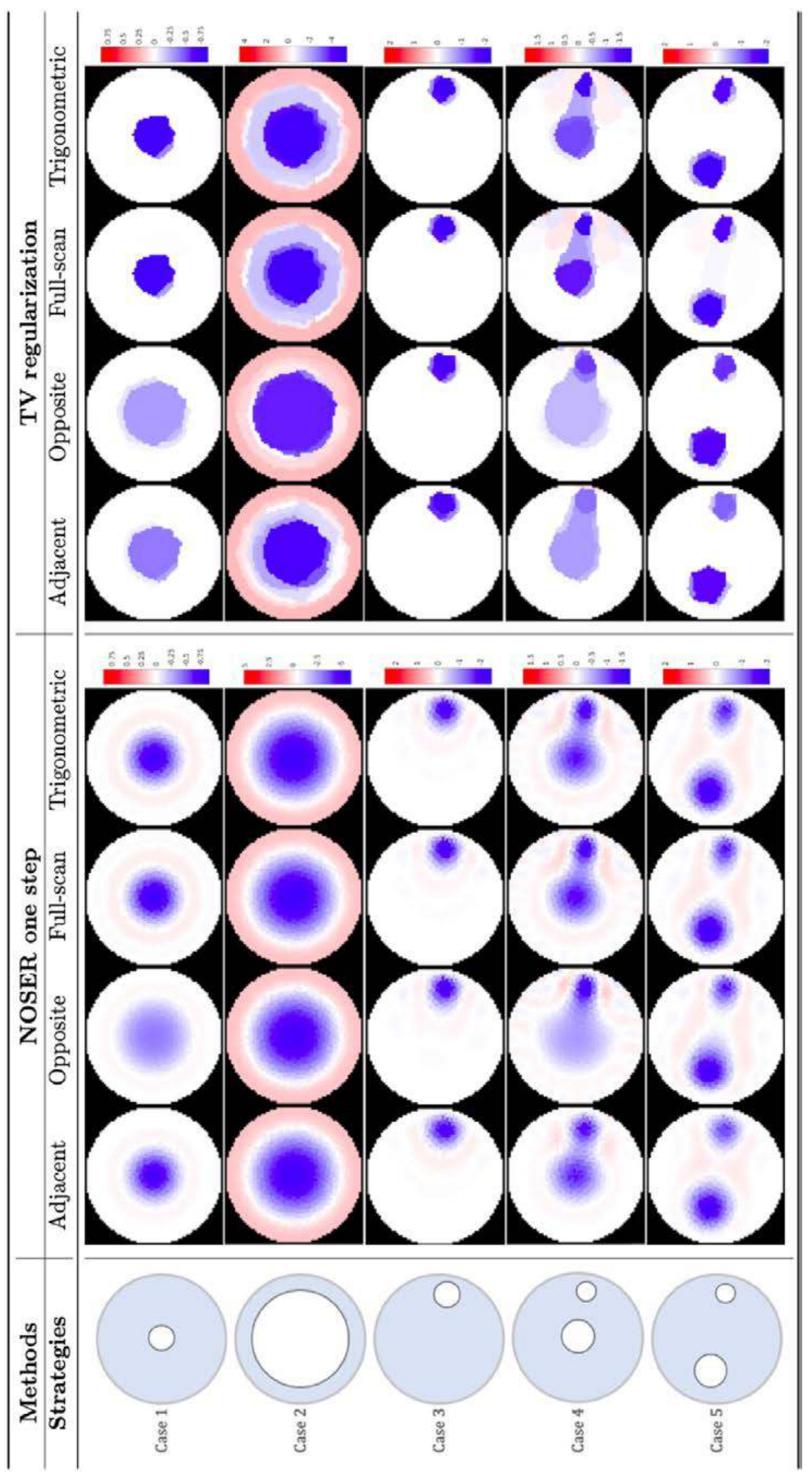

Figure 8. Reconstructions $\Delta \sigma_{\text {id }}^{h}$ of the conductivity contrast from synthetic measurements and using the NOSER method and TV regularization. 


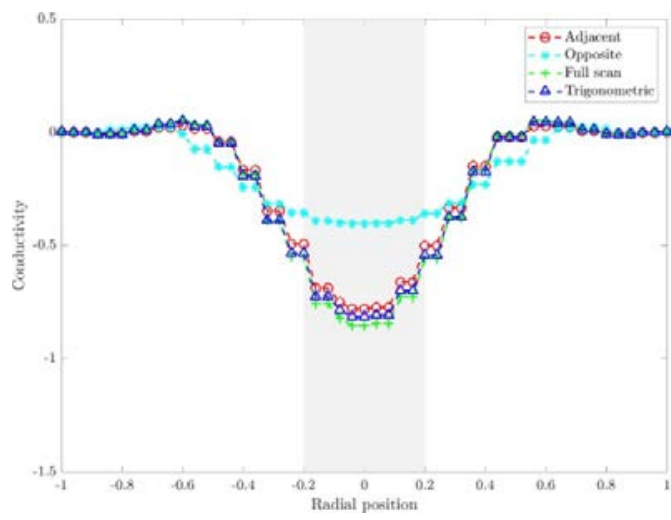

(a) NOSER method.

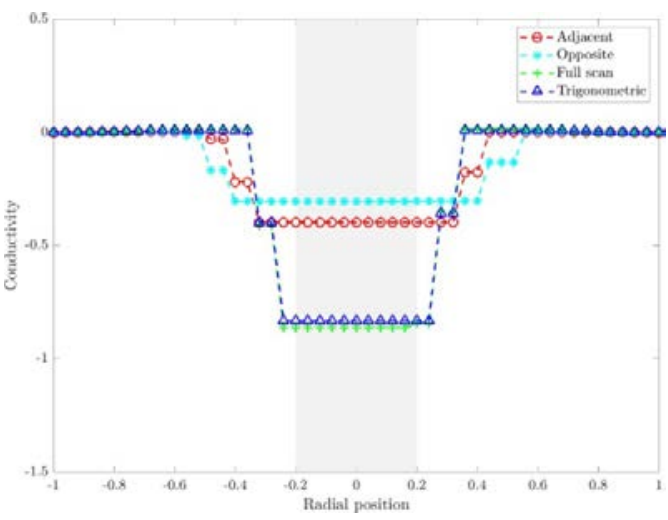

(b) TV regularization.

Figure 9. [Case 1] Profiles of the reconstructed conductivity contrasts $\Delta \boldsymbol{\sigma}_{\mathrm{id}}^{h}$ along one diameter for the different excitation strategies and reconstruction methods considered. Grey regions indicate the true objects.

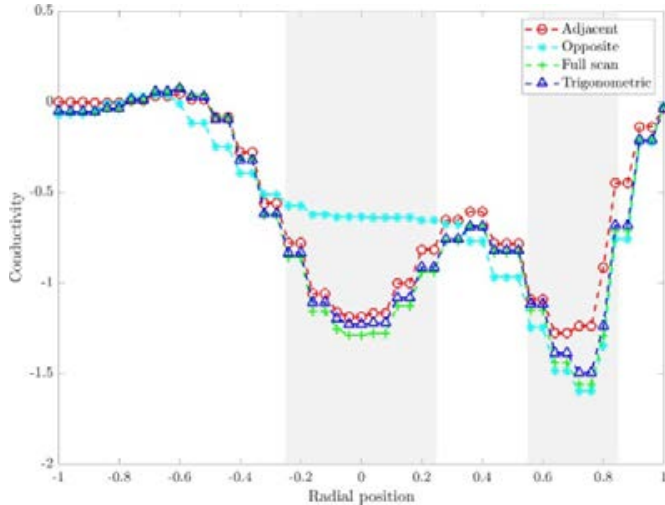

(a) NOSER method.

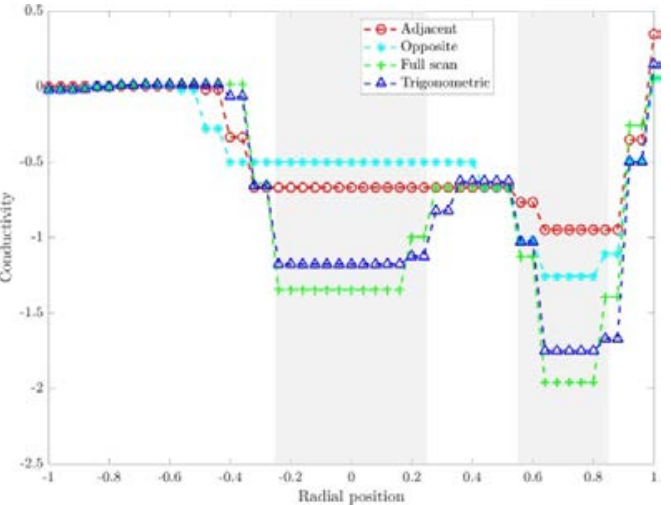

(b) TV regularization.

Figure 10. [Case 4] Profiles of the reconstructed conductivity contrasts $\Delta \boldsymbol{\sigma}_{\mathrm{id}}^{h}$ along one diameter for the different excitation strategies and reconstruction methods considered. Grey regions indicate the true objects.

case, the object(s) are correctly identified qualitatively, but their sizes is not systematically constrained quantitatively, see the cases 1, 2 and 4 . In terms of excitation strategy, the full-scan and trigonometric strategies seem to constrain the object(s) size(s) more than the others, but they may lead to artifacts in the reconstructions; see cases 4 and 5.

The profiles of the reconstructed conductivity distributions are extracted along one diameter and plotted in the figures 9 and 10 for the cases 1 and 4 respectively. The true objects to be reconstructed are indicated by the grey regions. These results highlight that the full-scan and trigonometric strategies lead systematically to images with higher conductivity contrasts compared to the others approaches. For Case 1, the opposite strategy leads to the least accurate reconstructions, in terms of object size and conductivity contrast, which agrees with the earlier statement that its resolution is limited by its relatively low number of independent measurements. It gives also unsatisfactory reconstructions in Case 4, with the concentric object being smooth out in the obtained image, thus making it difficult to discriminate the two objects. In comparison, the fullscan and trigonometric strategies give better reconstructions in Case 1, in terms of conductivity contrast and object size, as well as in Case 4, with the two objects being clearly distinguished.

4.2.2. Quantitative indicators. This section focuses on a quantitative evaluation of the quality of the reconstructed images of section 4.2.1. To do so, three criteria are introduced and evaluated on the obtained images.

4.2.2.1. Global identification error. An error indicator $\epsilon_{\mathrm{id}}$ is defined as the overall relative reconstruction error on the conductivity field. In the discrete setting considered, it is defined as follows:

$$
\begin{gathered}
\epsilon_{\mathrm{id}}=\frac{1}{A^{h}} \sum_{n_{e}=1}^{N_{\mathrm{el}}} \frac{\left|\boldsymbol{\sigma}_{\mathrm{id}}^{h}\left(n_{e}\right)-\boldsymbol{\sigma}_{\text {true }}^{h}\left(n_{e}\right)\right|}{\boldsymbol{\sigma}_{\text {true }}^{h}\left(n_{e}\right)} \boldsymbol{A}_{\mathrm{el}}^{h}\left(n_{e}\right), \\
\quad \text { with } \quad A^{h}=\sum_{n_{e}=1}^{N_{\mathrm{el}}} \boldsymbol{A}_{\mathrm{el}}^{h}\left(n_{e}\right),
\end{gathered}
$$

where the summation is performed over elements, whose total number is $N_{\mathrm{el}}$ and given their individual area $\boldsymbol{A}_{\mathrm{el}}^{h}\left(n_{e}\right)$. The 


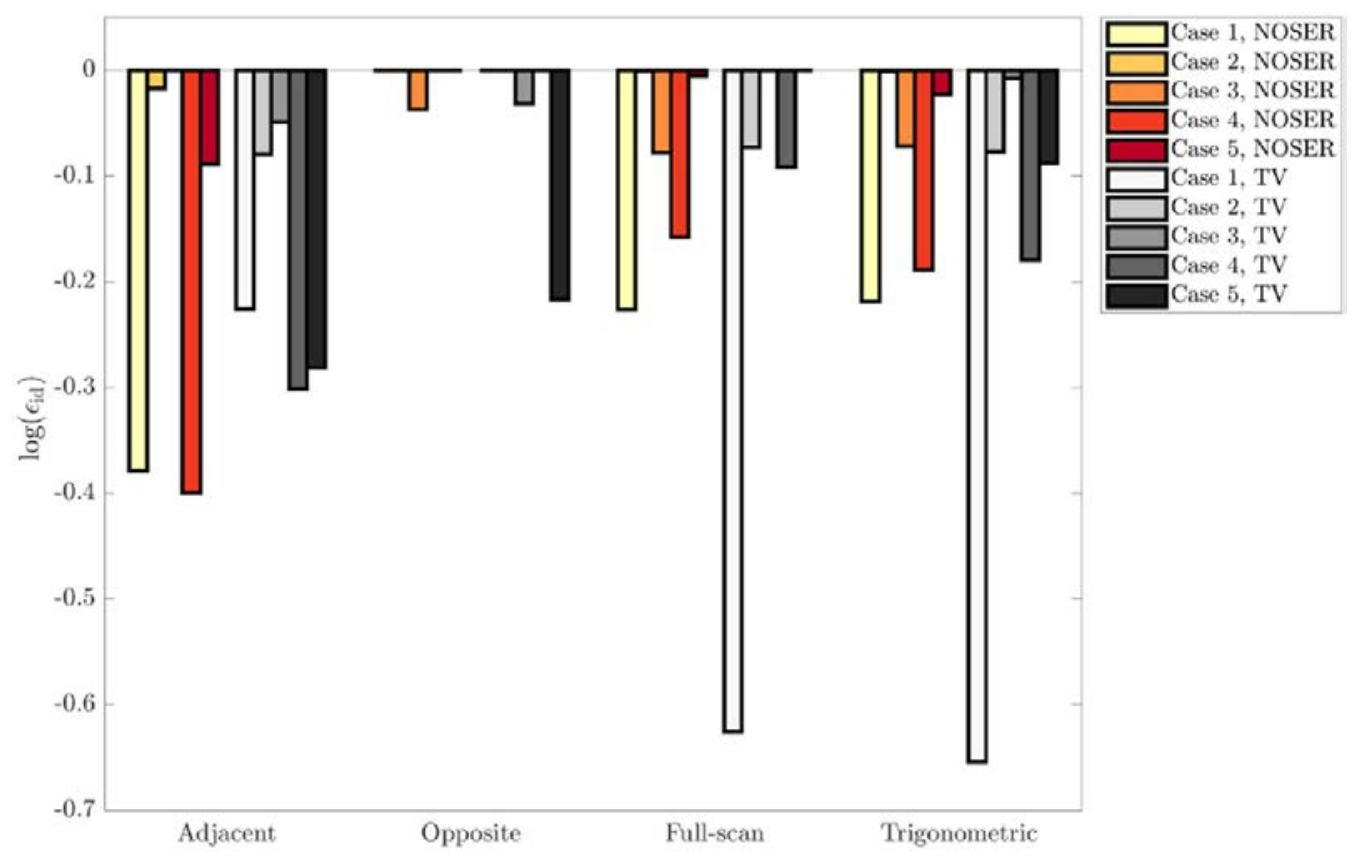

Figure 11. Logarithm of the global identification errors $\epsilon_{\mathrm{id}}$, normalized by the largest error among the four excitation strategies for each case and each reconstruction method. The value 0 is associated with the lowest-performing strategy and low values indicate relatively better performances.

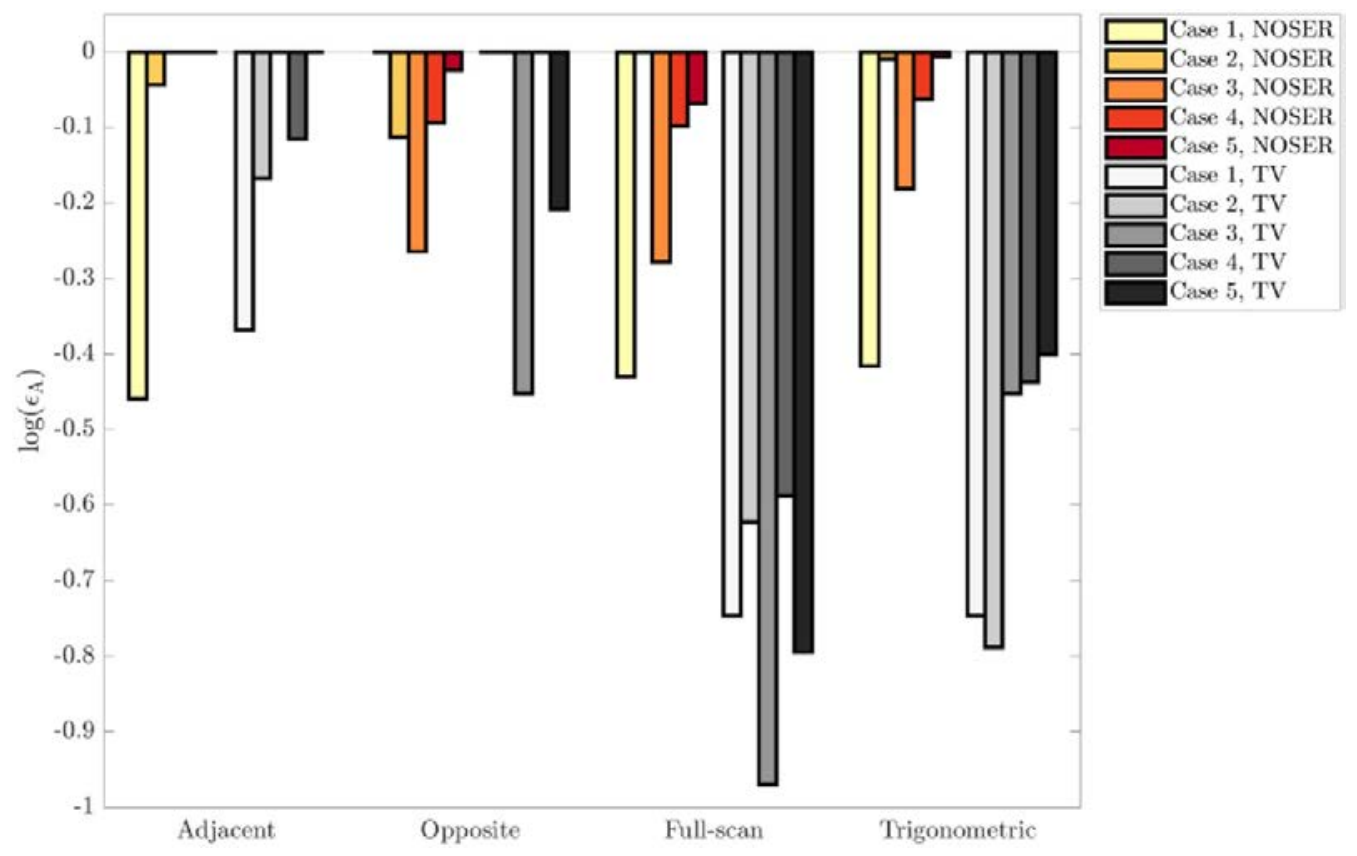

Figure 12. Logarithm of the geometrical area errors $\epsilon_{\mathrm{A}}$, normalized by the largest error among the four excitation strategies for each case and each reconstruction method. The value 0 is associated with the lowest-performing strategy and low values indicate relatively better performances.

indicator $\epsilon_{\text {id }}$ is computed for all of the reconstructed images of figure 8 . For each case and each reconstruction method, the values $\epsilon_{\text {id }}$ are normalized by the largest error among the four excitation strategies and their logarithm is then plotted in figure 11. In such a figure, low values indicate good relative performances.
The opposite strategy is almost systematically associated with the largest identification errors (with the normalized value 1 giving a zero logarithmic value). The adjacent method performs quite well on average while the full-scan and trigonometric strategies lead to comparable but lightly less accurate reconstructions overall. Yet, it should be noted that a 


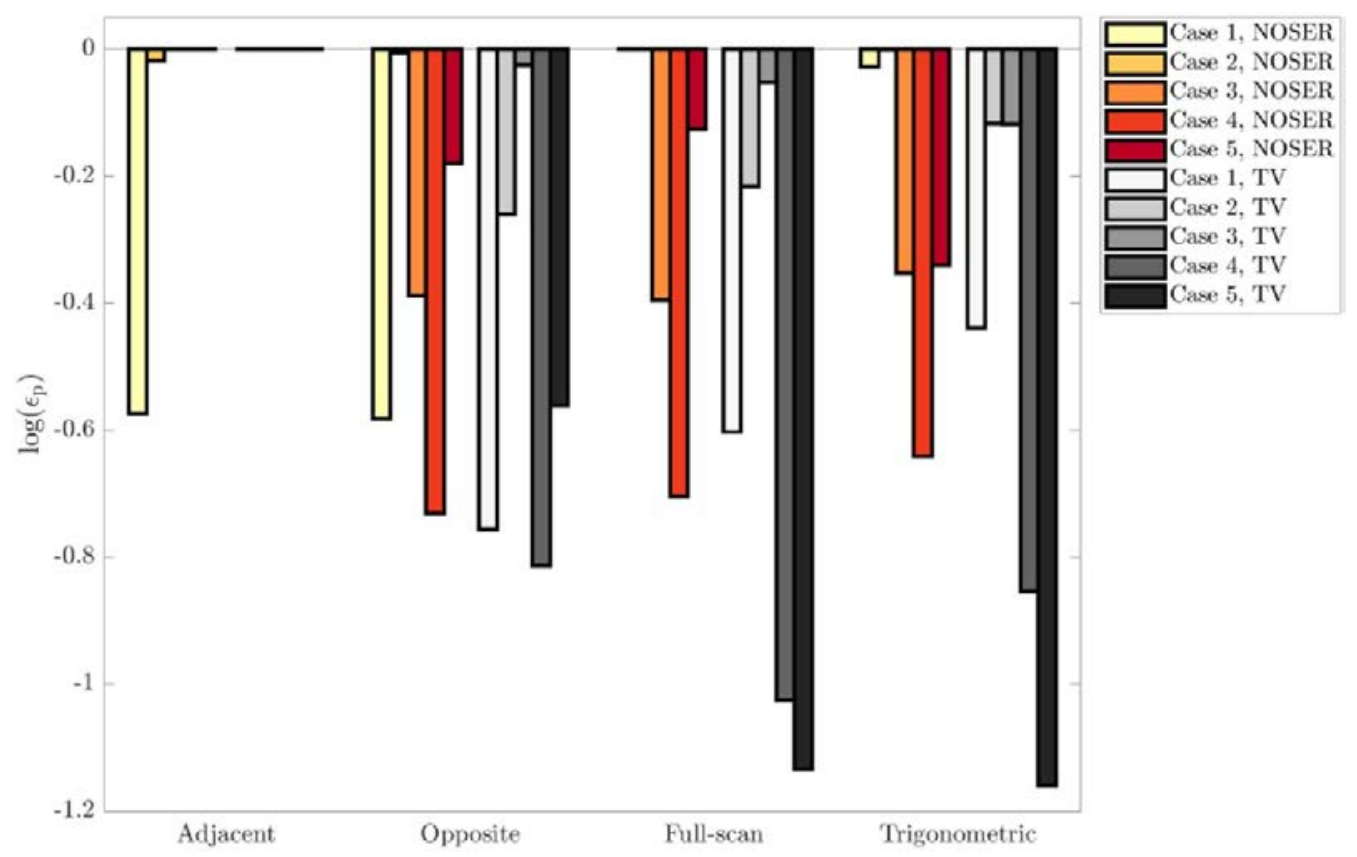

Figure 13. Logarithm of the positioning errors $\epsilon_{\mathrm{p}}$, normalized by the largest error among the four excitation strategies for each case and each reconstruction method. The value 0 is associated with the lowest-performing strategy and low values indicate relatively better performances.

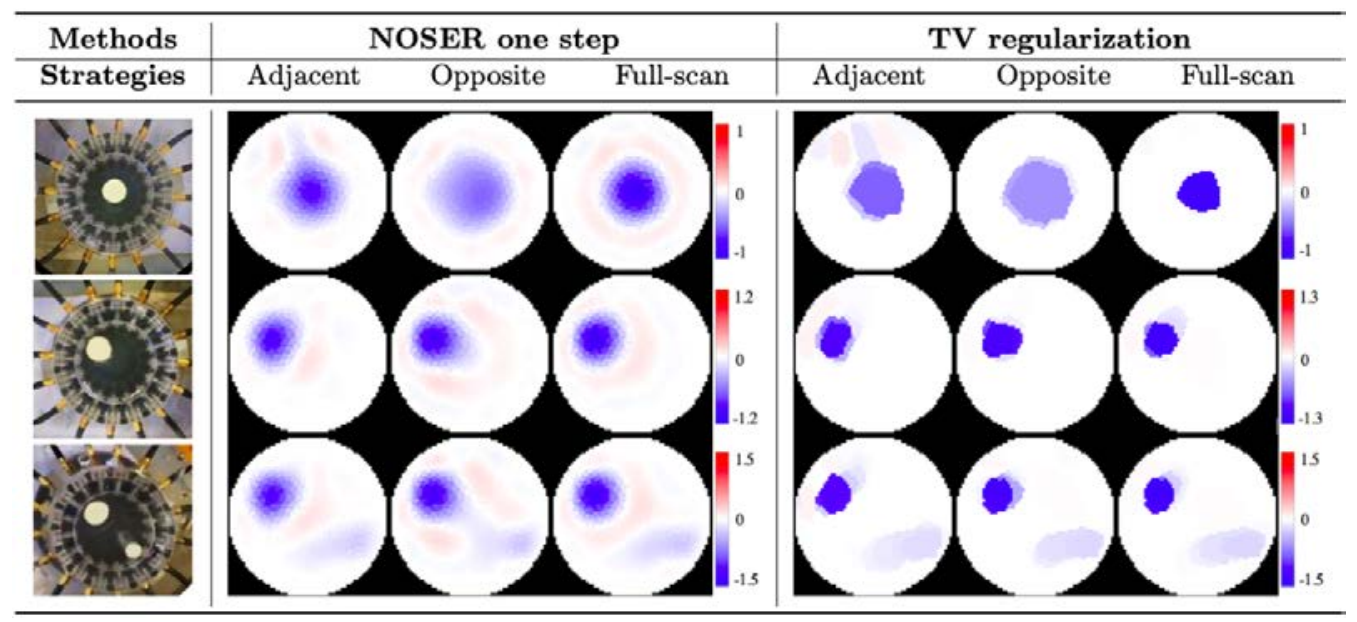

Figure 14. Reconstructions of the conductivity contrast $\Delta \boldsymbol{\sigma}_{\text {id }}^{h}$ from experimental data and using the NOSER method or TV regularization.

low value $\epsilon_{\text {id }}$ does not necessarily implies that the object(s) are correctly identified geometrically, see e.g. the reconstructions of figure 10(b). Therefore, one considers next two additional indicators of the geometrical errors on the reconstructed objects.

4.2.2.2. Geometrical area error. In EIT, the boundaries of highly contrasted objects are notoriously smooth out in reconstructed images. While TV regularization can remedy to that, in certain applications, such as two-phase flow imaging, the phase fraction is a critical parameter to estimate. In such situations, the reconstructed conductivity $\sigma_{\text {id }}^{h}$ can be further segmented into $\tilde{\boldsymbol{\sigma}}_{\text {id }}^{h}$ as follows:

$$
\tilde{\boldsymbol{\sigma}}_{\mathrm{id}}^{h}= \begin{cases}-1 & \text { if } \Delta \boldsymbol{\sigma}_{\mathrm{id}}^{h} \leqslant \frac{1}{4} \min \Delta \boldsymbol{\sigma}_{\mathrm{id}}^{h} \\ 0 & \text { otherwise }\end{cases}
$$

where $1 / 4$ is actually a user-chosen value. Doing so, a geometrical area error $\epsilon_{\mathrm{A}}$ is defined as

$$
\epsilon_{\mathrm{A}}=\frac{\left|A_{\text {id }}-A_{\text {true }}\right|}{A_{\text {true }}}
$$

where $A_{\text {id }}$ and $A_{\text {true }}$ are respectively the area of the object(s), i.e. the region(s) of lowest conductivity, in the segmented reconstruction and in the true image, respectively. The error indicator $\epsilon_{\mathrm{A}}$ is computed for all of the reconstructed images of 
figure 8. As done for the global identification error, normalization by the largest error among the four excitation strategies is employed for each case and each reconstruction method. Logarithmic values are then plotted in figure 12 .

According to this indicator, both the adjacent and opposite strategies perform poorly, on average, in identifying the phase fraction in the cases considered. In contrast, the performances of the full-scan and trigonometric strategies are relatively comparable to each other and significantly better than the former ones. This sheds a new light on the results of figure 11, as a low global identification error value is not necessarily associated with an inaccurate geometric reconstruction of the targeted object(s).

4.2.2.3. Positioning error. A third criterion is finally introduced to evaluate the accuracy of the object(s) position(s) in the reconstructed $\sigma_{\text {id }}^{h}$ distribution. As in some cases the objects connectedness can be difficult to assess in the reconstructed images, one focuses only on the objects barycenter. Doing so, one introduces the positioning error $\epsilon_{\mathrm{p}}$ as

$$
\epsilon_{\mathrm{p}}=\frac{\left|d_{\mathrm{id}}-d_{\text {true }}\right|}{D}
$$

with $d_{\text {id }}$ and $d_{\text {true }}$ being the distance of the object's barycenter to the origin in the reconstructed and true image, respectively, while $D$ is the diameter of the domain. As done with the two error indicators considered previously, normalization by the largest error among the four excitation strategies is employed for each case and each reconstruction method. Logarithmic values are then computed and the results are plotted in figure 13.

According to this third geometrical criterion, the adjacent strategy is associated with large systematic errors. The performances of the opposite, full-scan and trigonometric strategies are relatively comparable to each other. In some cases, the full-scan and trigonometric strategies seem to yield reconstructions that are slightly more accurate than the opposite strategy.

\subsection{Image reconstructions from experimental data}

In this last section, a number of image reconstructions from experimental data are provided. The experiments are static tests conducted in LTHC using the ProME- T system [11], see section 3.1. The system has 16 electrodes and a SNR of $50-60 \mathrm{~dB}$, from which the numerical results are evaluated with the noisy experimental data. Low conductive plastic rods are placed in a test section filled with salted water of conductivity $750 \mu \mathrm{S} \mathrm{cm}^{-1}$ to create the objective conductivity maps to be reconstructed. The system operates using the full-scan excitation strategy, which allows us to extract the measurement data for the adjacent and opposite strategies. Note that the implementation of the trigonometric strategy is currently beyond the technical capabilities of this system as it would require an upgrade with additional acquisition cards. Therefore, the latter strategy is not considered in this section, but the reader can refer to, e.g. [34, figure 11] and [35, figure 5.3], for typical reconstructed images from experimental data using the trigonometric strategy for configurations that are comparable to these considered here.

In the experimental setting, the test section has height of $250 \mathrm{~mm}$ and diameter of $51 \mathrm{~mm}$, while the size of electrodes is $5 \mathrm{~mm} \times 5 \mathrm{~mm}$. The static tests can therefore be considered as pseudo $2 \mathrm{D}$, which allows us to use the same reconstruction methods of the sections 4.1 and 4.2. The reconstructed images are provided in figure 14 for experimental configurations that correspond to the cases 1,3 and 5 of section 4.2. In combination with the quantitative results of section 4.2.2, the image reconstructions from experimental data tend to confirm qualitatively the preeminence of the full-scan strategy over the two other modes.

\section{Conclusions}

The adjacent, opposite, full-scan and trigonometric excitation strategies for EIT have been considered and compared in the light of a number of criteria, namely, complexity of a practical implementation, number of independent measurements, amplitude of the measured responses, sensitivity distribution and quality of the final reconstructed images. All of these excitation protocols are well within the reach of standard up-to-date hardware. Both numerical simulations and static experiments are employed for such a comparison.

Considering first the practical implementation criteria synthesized in section 3.5, it appears that the choice of a particular excitation strategy has to be closely related to specific practical constraints. For example, one might favor the quick response of the system rather than its amplitude or sensitivity distribution. Moreover, it should be noted that the practical criteria considered do not necessarily predict the imaging capabilities of a given excitation strategy. Therefore, this analysis is complemented by an evaluation of the quality of reconstructed images for the different excitation strategies, both from synthetic and experimental data. Five objective conductivity maps are considered and two different reconstruction methods are employed. For the studied configurations, the full-scan and trigonometric strategies lead to the best results on average, both qualitatively and quantitatively. They allow a better geometrical reconstruction of the object(s) geometry and yield images with sharper contrasts compared to the adjacent and opposite strategies.

To conclude, the simulations and experiments using artifacts discussed in this paper confirms the preeminence of the full-scan and trigonometric protocols with respect to the quality of the reconstructed images. Yet, they are associated with larger acquisition times or a system more complex compared to the adjacent and opposite strategies. Alme and Mylvaganam [36] reached similar conclusions for electrical capacitance tomography through comparing several measurement protocols numerically. These drawbacks can generally be circumvented using modern hardware and softwares implementations (e.g. FPGA and FDM) and sufficient computational 
power. Note finally that there exist alternative approaches to the imaging method, such as the eigenvalue-based approach of $[37,38]$ that allows to estimate the phase fraction from EIT data without image reconstruction.

\section{ORCID iDs}

Chunhui Dang (D) https://orcid.org/0000-0001-8798-8995 Mathieu Darnajou (D) https://orcid.org/0000-0003-4853-3693

\section{References}

[1] Ricciardi G, Pettigrew M and Mureithi N 2011 Fluidelastic instability in a normal triangular tube bundle subjected to air-water cross-flow J. Press. Vessel Technol. 13310

[2] Gisser D G, Isaacson D and Newell J C 1987 Current topics in impedance imaging Clin. Phys. Physiol. Meas. 839

[3] Cheney M and Isaacson D 1992 Distinguishability in impedance imaging IEEE Trans. Biomed. Eng. 39 852-60

[4] Lionheart W R B, Kaipio J and McLeod C N 2001 Generalized optimal current patterns and electrical safety in EIT Physiol. Meas. 2285

[5] Demidenko E, Hartov A, Soni N and Paulsen K D 2005 On optimal current patterns for electrical impedance tomography IEEE Trans. Biomed. Eng. 52 238-48

[6] Dickin F and Wang Mi 1996 Electrical resistance tomography for process applications Meas. Sci. Technol. 7247

[7] Sun J and Yang W 2015 A dual-modality electrical tomography sensor for measurement of gas-oil-water stratified flows Measurement 66 150-60

[8] Jia J, Wang H and Millington D 2017 Electrical resistance tomography sensor for highly conductive oil-water two-phase flow measurement IEEE Sens. J. 17 8224-33

[9] Adler A, Gaggero P O and Maimaitijiang Y 2011 Adjacent stimulation and measurement patterns considered harmful Physiol. Meas. 32731

[10] Dupré A, Ricciardi G and Bourennane S 2017 Novel approach for analysis and design of high-speed electrical impedance tomographic system for void fraction measurements in fast two-phase flows IEEE Sens. J. 17 4472-82

[11] Dupré A 2017 Electrical impedance tomography for void fraction measurements of harsh two-phase flows: prototype development and reconstruction techniques $P h D$ Thesis Ecole Centrale Marseille

[12] Stephenson D R, Mann R and York T A 2008 The sensitivity of reconstructed images and process engineering metrics to key choices in practical electrical impedance tomography Meas. Sci. Technol. 19094013

[13] Kauppinen P, Hyttinen J and Malmivuo J 2006 Sensitivity distribution visualizations of impedance tomography measurement strategies Int. J. Bioelectromagn. 8 1-9 (http://www.ijbem.org/volume8/number1/63-71.pdf)

[14] Kim M C, Kim S, Kim K Y and Lee Y J 2001 Regularization methods in electrical impedance tomography technique for the two-phase flow visualization Int. Commun. Heat Mass Transfer 28 773-82

[15] Kotze R, Adler A, Sutherland A and Deba C N 2019 Evaluation of electrical resistance tomography imaging algorithms to monitor settling slurry pipe flow Flow Meas. Instrum. 101572

[16] Wang M 2005 Electrode models in electrical impedance tomography J. Zhejiang Univ. Sci. A 6 1386-93
[17] Darnajou M, Dupré A, Dang C, Ricciardi G, Bourennane S and Bellis C 2019 On the implementation of simultaneous multi-frequency excitations and measurements for electrical impedance tomography Sensors 193679

[18] Wilkinson A J, Randall E W, Long T M and Collins A 2006 The design of an ERT system for 3D data acquisition and a quantitative evaluation of its performance Meas. Sci. Technol. 17 2088-96

[19] Sharifi M and Young B 2013 Electrical resistance tomography (ERT) applications to chemical engineering Chem. Eng. Res. Des. 91 1625-45

[20] NI PXIe 2016 Device Specifications NI 6368 X Series Data Acquisition (National Instrument)

[21] Santos S A, Robens A, Boehm A, Leonhardt S and Teichmann D 2016 System description and first application of an FPGA-based simultaneous multi-frequency electrical impedance tomography Sensors 161158

[22] Dupré A and Mylvaganam S 2018 A simultaneous and continuous excitation method for high-speed electrical impedance tomography with reduced transients and noise sensitivity Sensors 181013

[23] NI cRIO 2018 Device Specifications NI cRIO-9039: Embedded CompactRIO Controller with Real-Time Processor and Reconfigurable FPGA (National Instrument)

[24] Breckon W R and Pidcock M K 1988 Some mathematical aspects of electrical impedance tomography Mathematics and Computer Science in Medical Imaging (Berlin: Springer) pp 351-62

[25] Polydorides N and Lionheart W R B 2002 A Matlab toolkit for three-dimensional electrical impedance tomography: a contribution to the electrical impedance and diffuse optical reconstruction software project Meas. Sci. Technol. 131871

[26] Darnajou M 2020 A novel approach to high-speed electrical impedance tomography with frequency division multiplexing PhD Thesis Ecole Centrale Marseille

[27] Dang C 2020 Imaging and fast features extraction of two-phase flows using electrical impedance tomography PhD Thesis Ecole Centrale Marseille

[28] Sun J and Yang W 2014 Evaluation of fringe effect of electrical resistance tomography sensor Measurement 53 145-60

[29] Hecht F 2012 New development in FreeFem++ J. Numer. Math. 20 251-65

[30] Ma Y, Wang H, Xu L-A and Jiang C 1997 Simulation study of the electrode array used in an ERT system Chem. Eng. Sci. 52 2197-203

[31] Plonsey R and Collin R 1977 Electrode guarding in electrical impedance measurements of physiological systems-a critique Med. Biol. Eng. Comput. 15 519-27

[32] Cheney M, Isaacson D, Newell J C, Simske S and Goble J 1990 NOSER: an algorithm for solving the inverse conductivity problem Int. J. Imaging Syst. Technol. 2 66-75

[33] Borsic A, Graham B M, Adler A and Lionheart W R B 2009 In vivo impedance imaging with total variation regularization IEEE Trans. Med. Imaging 29 44-54

[34] Cook R D, Saulnier G J, Gisser D G, Goble J C, Newell J C and Isaacson D 1994 ACT3: a high-speed, high-precision electrical impedance tomograph IEEE Trans. Biomed. Eng. 41 713-22

[35] Newell J C et al 1993 In vivo impedance images using sinusoidal current patterns Clinical and Physiological Applications of Electrical Impedance Tomography pp 277-98

[36] Alme K J and Mylvaganam S 2007 Comparison of different measurement protocols in electrical capacitance tomography using simulations IEEE Trans. Instrum. Meas. $562119-30$ 
[37] Dang C, Darnajou M, Bellis C, Ricciardi G, Schmidt H and Bourennane S 2019 Numerical and experimental analysis of the correlation between EIT data eigenvalues and two-phase flow phase fraction Meas. Sci. Technol. 31015302
[38] Dang C, Darnajou M, Bellis C, Ricciardi G, Mylvaganam S and Bourennane S 2021 Improving EIT-based visualizations of two-phase flows using an eigenvalue correlation method IEEE Trans. Instrum. Meas. 70 1-9 